\title{
Herzinsuffizienz
}

\section{Eisenmangel verkürzt das Leben}

\section{Bei kardialen Patienten, insbesondere solchen mit Herzinsuffizienz, findet sich häufig ein Eisenmangel. Dieser verschlechtert nicht nur die Symptomatik, sondern verkürzt auch das Leben der Patienten.}

— $10-20 \%$ aller Herzinsuffizienzpatienten haben eine Anämie, und bei ca. $80 \%$ findet sich ein absoluter oder funktioneller Eisenmangel. Ursachen des absoluten Eisenmangels sind Mangelernährung, Malabsorption und chronischer Blutver-

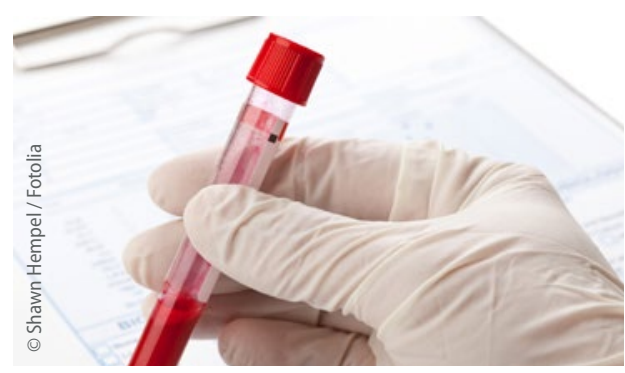

lust durch die antithrombotischen Medikamente. Der funktionelle Eisenmangel mit gestörter Eisenverfügbarkeit ist dagegen Folge der bei dieser Erkrankung häufigen chronischen Inflammation und der chronischen Niereninsuffizienz.

Dass eine Anämie bei herzinsuffizienten Patienten die Prognose quo ad vitam verschlechtert, ist gut belegt. In einer Studie stieg die 1-Jahres-Mortalität bei Vorliegen eines Eisenmangels von 65\% auf 73\%. Deshalb sollte bei allen Patienten mit einer systolischen Herzinsuffizienz mittels Bestimmung von Ferritin und evtl. der Transferrinsättigung nach einem Eisenmangel gefahndet werden.
Auch ohne Anämie nutzt Substitution In der FAIR-HF-Studie konnte gezeigt werden, dass eine Eisentherapie bei Patienten mit Eisenmangel den klinischen Status und die Lebensqualität deutlich verbessert, und zwar unabhängig vom $\mathrm{Hb}$-Wert. Eisenmangel hat bei Herzinsuffizienz einen doppelt ungünstigen Effekt auf den Sauerstoff-Stoffwechsel. So kommt es einmal zu einer funktionellen Beeinträchtigung aerober Enzyme und somit zu einer Störung der oxidativen Phosphorylierung, d.h. die $\mathrm{O}_{2}$-Nutzung ist beeinträchtigt. Zum andern vermindert ein niedriges $\mathrm{Hb}$ den $\mathrm{O}_{2}$-Transport. Beides beeinträchtigt die körperliche Leistungsfähigkeit, was durch eine Eisensubstitution verbessert werden kann.

Dr. Peter Stiefelhagen

- ESC-Kongress 2017 in München

\section{Eisenmangel wird häufig übersehen}

\section{Arbeiten deutsche Ärzte nicht leitlinienkonform?}

\section{In deutschen Kardiologie-Praxen werden Eisenmangel und Anämien bei Herzinsuffizienz-Patienten offensichtlich häufig übersehen, wie eine Registerstudie offenlegt.}

— Wie eine Auswertung des PrEP-Registers (Prävalenz des Eisenmangels bei Patienten mit Herzinsuffizienz) ergab, lag bei fast der Hälfte $(42,5 \%)$ der insgesamt 1.198 in dem Register eingeschlossenen Patienten mit chronischer Herzinsuffizienz ein Eisenmangel vor; definiert als Ferritinwert $<100 \mu \mathrm{g} / \mathrm{l}$ oder Ferritinwert von 100 bis $300 \mu \mathrm{g} / \mathrm{l}$ bei einer Transferrinsättigung $<20 \%$. Bei allen diesen Patienten war die Erkrankung im Vorfeld nicht bekannt. Eine Anämie ließ sich bei $18,9 \%$ der Patienten feststellen. Diese Erkrankung war vor Studieneinschluss gerade mal bei 4,8\% der Patienten diagnostiziert und wurde bei keinem einzigen durch eine Bluttransfusion oder Erythropoetin-Gabe behandelt.
„Trotz der hohen Prävalenz war der Eisenmangel somit bei allen PrEP-Teilnehmer unbekannt und die vorhandene Anämie blieb häufig unbeachtet", lautet das Fazit der Studienautoren um Dr. Stephan von Hähling, Universität Göttingen.

\section{Leitlinien auch in ambulanter Versorgung umsetzen}

Die Kardiologen plädieren daher dafür, die gültigen ESC-Leitlinien bzgl. einer Eisensubstitution auch in der ambulanten Versorgung umzusetzen. Darin wird eine solche Therapie für symptomatische Patienten mit chronisch stabiler Herzinsuffizienz und eingeschränkter Auswurffraktion empfohlen, die an einem Eisenmangel und einer Anämie lei- den, aber auch für Patienten, bei denen nur ein Eisenmangel vorliegt.

Eisenstatus routinemäßig bestimmen Darüber hinaus wird in den Leitlinien eine regelmäßige Bestimmung der Blutwerte nahegelegt, um reversible/behandelbare Ursachen der Herzinsuffizienz und Komorbiditäten - wie eben einen Eisenmangel - erfassen zu können. „Die Ergebnisse des PrEP-Registers liefern starke Argumente dafür, Eisenmangel und Anämien bei Herzinsuffizienz-Patienten auch in kardiologischen Praxen zu diagnostizieren und zu behandeln“, so die Autoren. Konkret bedeutet das, den Eisenstatus im ambulanten Setting routinemäßig zu bestimmen und ggf. eine Eisensupplementation als Therapie einzusetzen.

Veronika Schlimpert

- von Haehling S. et al. Clin Res Cardiol 2017; First Online: 22 February 2017. doi:10.1007/s00392-016-1073-y 